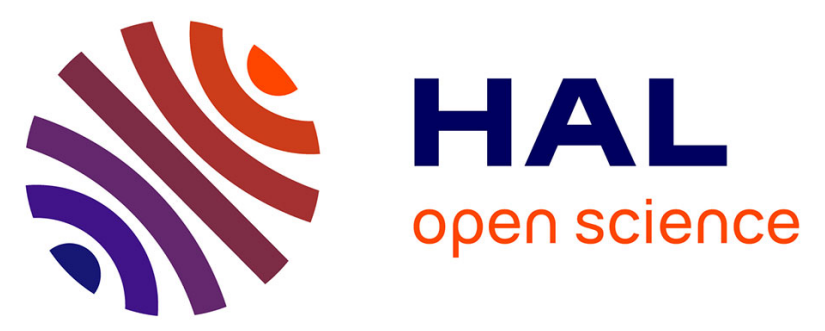

\title{
Une recomposition incertaine. Les élections présidentielles et législatives de 2002 en Languedoc-Roussillon
}

\author{
Paul Alliès, François Baraize, Emmanuel Négrier
}

\section{To cite this version:}

Paul Alliès, François Baraize, Emmanuel Négrier. Une recomposition incertaine. Les élections présidentielles et législatives de 2002 en Languedoc-Roussillon. Pôle Sud - Revue de science politique de l'Europe méridionale, 2002, Religion et politique, 17, pp.101-118. 10.3406/pole.2002.1288 . hal02488110

\section{HAL Id: hal-02488110 \\ https://hal.umontpellier.fr/hal-02488110}

Submitted on 28 Apr 2020

HAL is a multi-disciplinary open access archive for the deposit and dissemination of scientific research documents, whether they are published or not. The documents may come from teaching and research institutions in France or abroad, or from public or private research centers.
L'archive ouverte pluridisciplinaire HAL, est destinée au dépôt et à la diffusion de documents scientifiques de niveau recherche, publiés ou non, émanant des établissements d'enseignement et de recherche français ou étrangers, des laboratoires publics ou privés.

\section{(ㅇ)(1) $\$$}

Distributed under a Creative Commons Attribution - NonCommercial - NoDerivatives 44.0 


\section{Une recomposition incertaine. Les élections présidentielles et} législatives de 2002 en Languedoc-Roussillon

Mr Paul Alliès, Mr François Baraize, Mr Emmanuel Négrier

\section{Citer ce document / Cite this document :}

Alliès Paul, Baraize François, Négrier Emmanuel. Une recomposition incertaine. Les élections présidentielles et législatives de 2002 en Languedoc-Roussillon. In: Pôle Sud, n¹7, 2002. Religion et politique. pp. 101-118;

doi : https://doi.org/10.3406/pole.2002.1288

https://www.persee.fr/doc/pole_1262-1676_2002_num_17_1_1288

Fichier pdf généré le 23/04/2018 


\title{
Résumé
}

Le Languedoc-Roussillon est l'une des régions françaises où les scores d'extrême-droite ont été les plus élevés. C'est désormais une tradition inscrite sur une vingtaine d'années, une période qui est également marquée par de profonds bouleversements démographiques. Derrière l'établissement de ce courant politique, les forces politiques vivent des recompositions internes d'ampleur variable. La gauche, qui avait donné son nom au Midi Rouge, est en voie de moyennisation, perdant d'importants bastions électoraux, alors qu'elle avait conquis la presque totalité des sièges de députés, en 1997. La droite, marquée à l'échelle régionale par une stratégie d'alliance avec l'extrême-droite, a reconquis une majorité de sièges, en s'appuyant sur la naissance de l'UMP. Rien n'indique cependant que les rapports de force issus de ces deux scrutins, présidentiel et législatif, auront une durée plus grande que les deux précédentes photographies du Languedoc politique, blanc en 1993, rose- rouge en 1997. Une recomposition incertaine...

\begin{abstract}
Languedoc-Roussillon is one of the French regions where the scores of extreme-right were highest. It is from now on a tradition registered on a score of years, one period which is also marked by deep demographic upheavals. Behind the establishment of this political stream, the political forces had knew internal recombinings of variable width. The left, which had given its name to the Red South, is now closed to its national average, losing significant electoral bastions, whereas it had conquered almost the totality of the seats of deputies, in 1997. The Right, conser- vaticve and liberal parties, marked on a regional scale by a strategy of alliance with the extreme- right, reconquered a majority of seats, while being based on the birth of the UMP. Nothing states however that the power struggle resultingfrom these two polls, presidential and legislative, will have one duration larger than the two preceding photographs of political Languedoc, white in 1993, pink-red in 1997. A dubious recombining...
\end{abstract}




\title{
Une recomposition incertaine. \\ Les élections présidentielles \\ et législatives de 2002 \\ en Languedoc-Roussillon
}

\author{
Paul Alliès, François Baraize, Emmanuel Négrier

\section{RésumélAbstract}

Le Languedoc-Roussillon est l'une des régions françaises où les scores d'extrême-droite ont été les plus élevés. C'est désormais une tradition inscrite sur une vingtaine d'années, une période qui est également marquée par de profonds bouleversements démographiques. Derrière l'établissement de ce courant politique, les forces politiques vivent des recompositions internes d'ampleur variable. La gauche, qui avait donné son nom au Midi Rouge, est en voie de moyennisation, perdant d'importants bastions électoraux, alors qu'elle avait conquis la presque totalité des sìzges de députés, en 1997. La droite, marquée à l'échelle régionale par une stratégie d'alliance avec l'extrême-droite, a reconquis une majorité de sièges, en s'appuyant sur la naissance de l'UMP. Rien n'indique cependant que les rapports de force issus de ces deux scrutins, présidentiel et législatif, auront une durée plus grande que les deux précédentes photographies du Languedoc politique, blanc en 1993, roserouge en 1997. Une recomposition incertaine...

Languedoc-Roussillon is one of the French regions where the scores of extreme-right were bighest. It is from now on a tradition registered on a score of years, one period which is also marked by deep demographic upheavals. Behind the establishment of this political stream, the political forces had knew internal recombinings of variable width. The left, which had given its name to the Red South, is now closed to its national average, losing significant electoral bastions, whereas it had conquered almost the totality of the seats of deputies, in 1997. The Right, conservaticue and liberal parties, marked on a regional scale by a strategy of alliance with the extremeright, reconquered a majority of seats, while being based on the birth of the UMP. Nothing states however that the power struggle resulting from these two polls, presidential and legislative, will have one duration larger than the two preceding photographs of political Languedoc, white in 1993, pink-red in 1997. A dubious recombining...

\section{Mots-Clés/Keywords}

Abstentions, élections législatives, élections présidentielles, Languedoc-Roussillon, Abstention, Languedoc-Roussillon, legislative election, presidential election. 
Beaucoup de choses se sont passées dans ces quatre tours de scrutins majeurs du printemps 2002. D'abord ce que d'aucuns craignaient depuis longtemps: Jean-Marie Le Pen en tête du premier tour. Puis une gauche déboussolée par la perte de son leader, un électorat qui boude les urnes plus que d'habitude, et des appareils paralysés par la défaite du 21 avril. Jacques Blanc, adoubé patron de l'UMP, se proclamant rempart contre l'extrémisme, surfe sur une vague nationale et permet à une droite désormais unie de gagner deux sièges de députés sur trois. Derrière ces faits électoraux, la région continue de se transformer en accéléré. La croissance démographique ne faiblit pas, précipitant l'urbanisation du littoral, mais aussi de l'arrière-pays, rendant ainsi criante la nécessité de restructuration de l'armature urbaine comme de la rénovation du littoral touristique. Ce mouvement touche désormais d'autres aires urbaines que le bassin montpelliérain. Perpignan, Carcassonne, connaissent un nouveau dynamisme porté par la démographie. Au nord, pourtant, la restructuration du bassin gardois est toujours incertaine.

Dans cette société en recomposition, les comportements électoraux continuent de trahir les signes de la déstructuration en cours.
Ainsi, le corps électoral augmente toujours bien moins vite que la population. Pas seulement du fait d'une hausse de la natalité (qui commence à être sensible), mais aussi par une absence tendancielle de ré-inscription sur les listes électorales d'une partie des nouveaux arrivants. L'abstention du premier tour des présidentielles touche un électeur sur quatre dans l'ensemble de la région, un électeur sur trois dans une majeure partie des grandes villes. Si elle a faibli au second tour, les votes blanc et nuls ont pris le relais, passant régionalement de $2,3 \%$ des inscrits au premier tour à 5,6\% au second. Le mouvement s'est amplifié aux législatives, pour atteindre un record historique de $36 \%$ d'abstentions au premier tour, de $38 \%$ au second. À ce niveau, la désaffection des urnes contribue non seulement à bouleverser les données traditionnelles du vote, comme le montrent les résultats de la gauche, mais posent encore une fois la question lancinante de l'adéquation de l'offre politique à son territoire.

Le Languedoc-Roussillon entame une nouvelle phase de sa restructuration politique. La gauche urbaine des années 80 marque dangereusement le pas, la droite profite de sa dynamique d'union, le comportement des électeurs FN reste toujours aussi erratique. 
Aussi est-il plus que jamais difficile de lire les évolutions prochaines sans replacer les mouvements de 2002 dans leurs trajectoires historiques récentes. C'est ce que nous proposons de faire ici, en examinant principalement les trois grands blocs que constituent ce que fut la "gauche plurielle", la droite unie et l'archipel d'extrême-droite.

\section{Le grand reflux de la gauche}

C'est devenu un grand classique journalistique: chaque élection nationale est l'occasion d'une annonce irrémédiablement historique, celle de "la chute finale du Midi rouge" (Le Figaro, 23/04/02), du "Midi rouge (qui) vire au brun" (Le Monde, 23/04/02), du "Midi qui n'est plus rouge" (Midi Libre, 22/04/02). Pourtant, c'est dès le lancement de cette revue, il y a bientôt dix ans, que nous y analysions "un midi en voie de banalisation politique" (Alliès, 1995). Sans qu'il soit nécessaire de mobiliser, pour l'instant, les subtilités de la théorie des réalignements (Martin, 2000), rappelons que les années 1981-84 ont ouvert un cycle brutal et durable de mutation des comportements électoraux, en particulier en Languedoc-Roussillon. Les changements à l'œuvre depuis, affectent les équilibres électoraux mais aussi le système partisan, les politiques publiques et les rapports que les citoyens entretiennent avec la politique en général. Ainsi le rapport de force droitegauche s'est-il globalement équilibré dans cette région, mais il s'est aussi structurellement transformé au sein de chaque "camp". La permanence de l'offre politique (le sigle des partis, le personnel candidat) masque ce phénomène que le calendrier électoral et ses alternances se chargent régulièrement de réitérer. Celui de ce printemps 2002 l'a fait sans révéler de nouveaux bouleversements: la gauche n'est plus "culturellement" majoritaire dans le Midi, sa géographie électorale est tendanciellement régressive, sa recomposition est problématique.

\section{Le signal des présidentielles}

Le premier tour de ces élections n'est qu'une confirmation du reflux linéaire de la gauche amorcé en 1981. Jusque-là Mitterrand $y$ atteint les 50,51\% (en 1974). Puis les deux candidats PS et PC passent à $48,15 \%$ en $1981,41,57 \%$ en $1988,35,25 \%$ en 1995 . Le 21 avril 2002 , ils sont à $26,94 \%$. Il faut les $5,2 \%$ de Chevènement, les $4,76 \%$ de Mamère, les $1,78 \%$ de Taubira pour combler péniblement le déficit. Et ce décompte, discutable car annexant trop simplement des marges jusqu'ici superflues, ne règle en rien le problème de fond: celui de l'érosion confirmée des bastions qui faisaient la force de la gauche régionale tout entière. Deux cas suffisent a l'illustrer, celui du Parti Communiste dans le Gard et du Parti Socialiste dans l'Aude. Dans le Gard, le PC en est arrivé au stade ultime de l'effondrement. Il perd plus de la moitié de ses électeurs par rapport au scrutin de 1995 ; ils ne sont plus que 5,26\%. À Alès, Robert Hue passe de $18,51 \%$ à 9,17\%. Dans l'Aude, Lionel Jospin fait certes cinq points de plus que sa moyenne nationale dans le seul département de la région où il est en tête devant tous les autres candidats (il l'était dans quatre départements sur cinq en 1995). Mais il a perdu près de dix points depuis le $1^{\circ}$ tour de 1995. Et c'est Jean-Marie Le Pen qui progresse le plus (près de sept points) en se hissant au deuxième rang des candidats.

Ces deux exemples signalent l'evasion d'un électorat populaire, en partie vers l'extrêmedroite, conformément aux reclassements sociologiques nationaux. Même en Lozère, Jospin perd huit points et recule dans les 
Cévennes traditionnellement socialistes. Le Pen atteint près de $15 \%$ dans le canton de Barre-Les-Cévennes (24,8 \% au Mas Gros), vieux fief de gauche. Par contraste, la résistance d'une ville comme Montpellier où Jospin est en tête et ne perd qu'un peu plus de sept points par rapport à 95, alors que Le Pen ne gagne qu'un point, confirme l'assise privilégiée du PS dans le haut des couches moyennes urbaines.

Une autre partie de cet électorat a donc fuit vers l'extrême-gauche. Pour autant celle-ci réalise un score inférieur de un point à sa moyenne nationale $(10,44 \%)$. A Alès comme à Carcassonne elle ne dépasse pas les $9 \%$. C'est à Montpellier qu'elle fait un de ses meilleurs résultats $(9,7 \%)$ avec une structure sociologiquement significative : Olivier Besancenot y fait quasiment jeu égal avec Arlette Laguillier, confirmant ainsi le profil contrasté de son public au niveau national (jeune, diplômé, actif). Cette extrêmegauche, si elle apparaît comme numériquement en progression, reste socialement hétérogène. De plus elle est territorialement dispersée, réalisant souvent des chiffres élevés dans de toutes petites communes. Elle n'apparaît donc pas comme le relais naturel de la gauche gouvernementale: elle n'a ni bastion significatif en voie de formation, ni élus, ni d'identité sociale comparable à celle, traditionnelle, de la gauche parmi la petite-bourgeoisie agraire ou d'État.

Le premier tour des présidentielles était donc bien un nouveau signal dans ce parcours déjà ancien des réalignements électoraux méridionaux. La gauche dans son ensemble y est toujours plus sensible à la conjoncture électorale nationale, pouvant régresser ainsi fortement d'un scrutin l'autre. Dans les Pyrénées-Orientales, Lionel Jospin passe de la première à la troisième place entre 1995 et 2002, victime d'une abstention record. Aucun reclassement stable de l'électorat n'est régionalement perceptible et le caractère ultra-nationalisé de l'élection accentue ce phénomène. $\mathrm{Ni}$ le néo-radicalisme de Taubira, ni l'antienne républicanisme de Chevènement n'ont offert une quelconque alternative puisque tous deux sont restés en-deçà de leur moyenne nationale $(1,78 \%$ pour l'une et $5,20 \%$ pour l'autre). Tout se passe donc comme si la gauche ne devait ses bons résultats qu'à l'enracinement local de ses élus et au vote utile ou par défaut en faveur du seul Parti Socialiste. C'est ce qu'allaient pouvoir confirmer les législatives.

\section{La "moyennisation" de la gauche}

La banalisation électorale du LanguedocRoussillon a accompagné ce qui apparaît bien comme un déclin de la gauche à l'occasion des élections nationales et vis-d-vis d'une histoire désormais révolue. Cette banalisation et ce déclin apparaissent nettement à travers la succession des quatre dernières élections législatives $(1988,1993,1997,2002)$ qui ont donné les résultats nationaux que l'on sait: courte victoire de la gauche en 88 , déroute en 93, succès en 97 et échec relatif en 2002. Dans ce jeu de balancier les circonscriptions législatives se répartissent en trois catégories:

(i) Celles où le cumul des gauches est majoritaire. La région n'en compte qu' une: la 4' des Pyrénées-Orientales. Et celles qui sont plutôt à gauche (c'est-à-dire ont voté à gauche trois fois sur quatre). La région en compte six: trois dans l'Aude, deux dans l'Hérault (la $5^{c}$ et la 7c), une dans le Gard (la 5).

(ii) Celles qui sont toujours à droite. Il n'y en a qu'une, en Lozère. Et celles qui sont plutôt à droite (selon le même ratio). Il y en a six: deux dans les P. O. (la $1^{\text {ec }}$ et la $3^{c}$ ) deux dans $l^{\prime H e ́ r a u l t ~(l a ~} 1^{\text {re }}$ et la $3^{\mathrm{e}}$ ), une dans le Gard (la $\left.1^{\mathrm{r}}\right)$, et une en Lozère (la $\left.1^{\mathrm{r}}\right)$. Une autre (la $4^{\mathrm{e}}$ 
de l'Hérault) a basculé à droite après avoir été de gauche en 88, 93 et 97.

(iii) Celles qui sont opportunistes, c'est-àdire qui votent dans le sens du vainqueur national du scrutin. Il y en a six: la $2^{\circ}$ des P. $O$., la $6^{e}$ et la $2^{e}$ de l'Hérault, les $2^{e}, 3^{c}$ et $4^{e}$ du Gard.

On voit nettement dans cet ensemble évolutif la gauche perdre régulièrement du terrain même si, au total, le rapport de forces en nombre de députés est tout à fait conforme à la tendance nationale, avec un léger avantage pour la gauche, lequel se réduit d'élection en élection du fait de la part prise par les circonscriptions opportunistes: 14 députés PS et un PC en 88 (contre 9 pour la droite); 3 députés en 93; 20 députés en 95 et 6 en 2002. Mais l'équilibre presque parfait de la répartition de ces circonscriptions en trois tiers, recouvre une réalité bien plus complexe.

D'abord, l'amplitude plus favorable à la gauche qu'à la droite, notamment en 1997, était due au nombre des triangulaires imposées par le Front National alors (sept au total). En 2002, le fléchissement de l'extrême-droite au premier tour a réduit celles-ci à quatre (à Bagnols, Beaucaire, Sète et Béziers). Son élimination dans des départements où elle était traditionnellement très puissante, comme les P. O. est directement responsable de la perte par la gauche de trois des quatre circonscriptions catalanes. Cela est vrai aussi dans les deux circonscriptions de l'est de l'Hérault et dans deux du Gard. Lors de ce premier tour en effet, à l'exception de l'Aude où les candidats du FN ont enregistré une légère progression par rapport à 97, partout ailleurs et surtout dans leurs zones de force, ils ont fortement reculé. Leur électorat, trop bien habitué aux allers et retours avec la droite régionale grâce aux accords passés avec Jacques Blanc, a voté utile dès le premier tour des législatives. L'électorat frontiste est, bien plus que celui de la France septentrionale qui revient plus volontiers vers la gauche au second tour, en voie de radicalisation droitière même dans sa base populaire. La gauche ici en fait indirectement et irrémédiablement les frais.

La gauche, mais pas toute entière. Les candidats du PS, tous sortants, améliorent leurs scores de 97 de 1 à 9,5 points (la $2^{e}$ des $P$. O.) dans plus de la moitié des circonscriptions (11 sur 21) à offre partisane constante. Ils le font au détriment de leurs partenaires de la gauche plurielle et de leurs censeurs d'extrêmegauche. Ces derniers retrouvent des scores très faibles comme à l'accoutumée (entre $1,6 \%$ et 4,5). Une bonne partie de leurs électeurs des présidentielles sont rentrés au bercail de la gauche traditionnelle ou se sont abstenus. Quant aux Verts, ils ne réussissent nulle part à imposer un chiffre significatif sauf à Montpellier-sud (6,6\%) et à Montpellier-Lodève $(13,45 \%)$. Partout ailleurs leur étiage se situe à $1,8 \%$. Si on totalise les résultats des élections présidentielles, législatives et européennes à compter de la candidature R. Dumont en 1974 (soit neuf élections), ceux-ci présentent une remarquable stabilité dans la stagnation et dans la cartographie: une fourchette entre 0,85 et $3,08 \%$ (hormis le pic exceptionnel des européennes de 1999), toujours nettement en-dessous de leur moyenne nationale, avec un enracinement privilégié qui n'a pas varié durant 25 ans, dans l'est de l'Hérault. Mais c'est le Parti Communiste qui, lui régresse de manière linéaire et fait le plus les frais de la rupture, dans le comportement des électeurs de gauche, d'une tactique d'union d'une circonscription à l'autre. Il avait accepté de retirer ses candidats dans huit circonscriptions pour espérer mobiliser toute la gauche autour de ses quatre députés sortants. Un seul arrivera vainqueur au second tour à la faveur d'une triangulaire (François 
Liberti à Sète) mais au prix d'une chute impressionnante de ses voix par rapport à 97 (moins $4,4 \%$ au premier tour et moins $18,46 \%$ au second).

Au total, la gauche du Languedoc-Roussillon se trouvait au soir du premier tour et par rapport aux 22 régions françaises, dans une position très moyenne: au $11^{\mathrm{e}}$ rang du total des voix de la gauche plurielle $(38,1 \%)$; au $9^{c}$ rang de celui du seul PS (26,9\%); l'extrême gauche y est au $14^{\mathrm{c}}$ rang $(2,8 \%)$. Rapportés à ceux de la droite gouvernementale $(35,2 \%)$ qui classent celle-ci à l'avant dernier rang interrégional dans sa catégorie, ces chiffres ne sont pas si mauvais. Ils restent toutefois insuffisants pour garantir une recomposition et sauvegarder un rapport de forces avec l'ensemble de la droite dans la deuxième région française d'extrême-droite $(18,1 \%)$. C'est ce que révélait clairement le second tour. Le nombre et l'amplitude des pertes des candidats de gauche par rapport à leurs scores de 97 dressent le tableau d'un vrai glissement de terrain. Seuls quatre candidats gagnent des voix.

\begin{tabular}{llll}
\hline $1^{*}$ & J. CODOGNES - PS & P.O. & $+4,17 \%$ \\
$2^{\circ}$ & A. CLARY - PC & Gard $+1,90 \%$ \\
$3^{\circ}$ & J. VILA - PC & P. O. $+1,74 \%$ \\
$4^{\circ}$ & G. ROSEAU -PS & Hérault $+1,50 \%$ \\
\hline
\end{tabular}

On observera que les deux candidats PC "gagneurs" bénéficiaient du soutien du PS dès le premier tour. L'union avec les socialistes est visiblement le seul moyen pour ce parti de maintenir le score de ses candidats à un bon niveau. Quant aux deux socialistes, ils ont en commun d'être plutôt marginaux par rapport aux fédérations départementales de leur parti et de ne cumuler aucun mandat significatif. Les quinze autres candidats reculent, mais de manière inégale:

\begin{tabular}{rlll}
\hline $1^{\text {er }}$ & F. LIBERTI - PC & Hérault & $-18,46 \%$ \\
$2^{\circ}$ & C. LAZERGES - PS & Hérault $-12,94 \%$ \\
$3^{\circ}$ & H. COLAS - PS & Hérault & $-11,30 \%$ \\
$4^{\circ}$ & P. MALAVIELLE PC & Gard & $-9,88 \%$ \\
$5^{\circ}$ & A. BARRAU - PS & Hérault & $-6,73 \%$ \\
$6^{\circ}$ & K. MESQUIDA - PS & Hérault & $-6,43 \%$ \\
$7^{\circ}$ & G. REVOL - PS & Gard & $-6,06 \%$ \\
$8^{\circ}$ & H. SICRE - PS & P.O. & $-6,07 \%$ \\
$9^{\circ}$ & J. BASCOU - PS & Aude & $-5,69 \%$ \\
$1^{\circ}$ & J.C. CHAZAL-PS & Lozère & $-5,53 \%$ \\
$11^{\circ}$ & J.C. PEREZ - PS & Aude & $-5,25 \%$ \\
$12^{\circ}$ & C. BOURQUIN - PS & P.O. & $-4,96 \%$ \\
$13^{\circ}$ & A. FABRE-PUJOLS & Gard & $-4,41 \%$ \\
$14^{\circ}$ & G. FRECHE - PS & Hérault & $-4,08 \%$ \\
$15^{\circ}$ & D. ALARY-PS & Gard & $-3,95 \%$ \\
\hline & & &
\end{tabular}

Compte tenu des spécificités propres à chaque circonscription, c'est dans l'Hérault que la régression est la plus massive (à l'exception de G. Frêche) même si elle est parfois masquée par des victoires singulières (celle de K. Mesquida, finalement seul député socialiste de ce département et de F. Liberti, seul député communiste de la région). D'évidence ni "la prime au sortant", ni les tactiques de premier tour (union ou division) ne suffisent pour combattre la régression tendancielle des personnalités en course. Par contre, force est de constater que parmi les "moins perdants" figurent les élus cumulant leur mandat de député sortant avec celui d'un exécutif local d'importance (présidence de Conseil Général du Gard, des P. O., et mairie/agglomération de Montpellier). Mais plus que l'explication par la personnalité des candidats, toujours tentante dans un scrutin uninominal, cette régression globale témoigne d'une incapacité de la gauche régionale d'organiser localement et d'unifier durablement ses électorats de plus en plus fragmentés. Ceux-ci connaissent une vraie mutation, repérable à travers la géographie électorale. 
Un réalignement structurel

La théorie des réalignements déjà mentionnée, distingue trois niveaux dans les évolutions électorales.

(i) Un niveau de très long terme, de l'ordre d'au moins une génération. Il concerne la disparition ou la formation de groupes sociaux, la migration des populations, les changements de pratiques religieuses. Le Languedoc-Roussillon est très caractéristique de cette mutation de long terme. Ce que nous analysions dans la comparaison des élections régionales de 1992 et 98 reste intégralement valable: le changement social et les dynamiques électorales se nourrissent l'un l'autre. Une urbanisation balnéaire et en grappe, une polarisation économique et sociale où s'imposent les professions intermédiaires et le salariat instable produisent des formes récurrentes d'atomisation et d'anomie sociale. C'est à cela qu'est due en profondeur la disparition de l'identité du "Midi Rouge".

(ii) Le réalignement a besoin d'un deuxième niveau, celui de la conjoncture, où opèrent des facteurs qui alimentent le mouvement du balancier droite-gauche le temps d'une élection. La situation économique, l'impact local d'une élection nationale (ici l'échec de Lionel Jospin aux présidentielles), un événement international (le 11 septembre et son cortège de peurs) peuvent agir fortement sur le comportement des électeurs indépendamment de leur proximité avec des forces politiques. Ces deux niveaux ont été bien analysés par la science politique.

(iii) Le troisième l'a été beaucoup moins. C'est celui du moyen terme, où interagissent les deux premiers: le niveau de la modification de la structure des systèmes partisans. Il est le terrain où s'organise une rupture entre des aspirations nouvelles de l'électorat et le système partisan qui a tendance à perpétuer les clivages sur lesquels il s'est structuré lors du précédent réalignement. Ce niveau est généralement occulté par l'offre politique, tellement l'opposition gauche/droite est elle-même une contrainte structurelle de la vie politique. Et ce n'est pas cette opposition qui est en cause (elle est inhérente au bon fonctionnement de toute démocratie représentative), mais bien plutôt le contenu de chacun de ses termes: l'existence d'un leadership sur le territoire de l'élection, le degré de dispersion des candidatures, le fonctionnement des alliances, la nature (complexe ou simple) du choix offert, voilà autant de facteurs qui agissent à moyen terme. Il est très facile de démontrer combien ce niveau intermédiaire joue, depuis le milieu des années quatre-vingt, en défaveur de la gauche en Languedoc-Roussillon et diamétralement, en faveur de la droite régionale. L'impact des alternances gouvernementales, l'importance prise par la question de l'immigration puis de l'insécurité, l'échec répété de la construction d'un leadership de gauche à l'échelle régionale, le repli corrélatif du Parti Socialiste sur l'institution départementale (dès lors qu'il contrôle quatre Conseils Généraux sur les cinq que compte une Région avec laquelle il ne peut et ne veut collaborer pour des raisons politiques), son incapacité à réduire la fragmentation politique plus forte et plus constante ici qu'ailleurs, voilà la liste non-exhaustive de ce qui mine l'ordre électoral de la gauche. Du coup, il est peu surprenant d'observer d'élection en élection le décrochage sociologique de son électorat.

Outre la dilution de ses bastions, déjà notée, la gauche est surtout forte dans les zones rurales de l'Aude, du Vallespir, de l'arrière-pays héraultais, des Cévennes gardoises. Là, elle parvient souvent à récupérer l'électorat "chasseur" et environnementaliste. Là, une population vieillie assure, par sa loyauté politique, et par "contagion", une reproduction de 
comportements auprès de nouveaux arrivants. Mais la gauche ne parvient pas à conquérir ou reconquérir les villes et s'affaisse dans les zones périurbaines, toutes offertes à la "droite de courtoisie" ou à l'extrême-droite protestataire. Le contrôle de la seule capitale régionale, au statut d'ailleurs contesté et qui n'a toujours pas, malgré sa remarquable croissance, la taille critique (comme Marseille ou Toulouse) pour exercer une influence sur sa grande périphérie, souligne l'extranéité régionale du socle social et culturel de cette gauche des couches supérieures nouvelles. Tout se passe dans ce réalignement comme si l'électorat identifiait de plus en plus l'implantation des partis avec la réalité du pouvoir sur le territoire. La définition et la conduite de politiques publiques appellent un seuil de visibilité en deçà duquel leur attribution à une autorité politique est difficile ou impossible pour les populations concernées. La complexification de la décentralisation, l'enchevêtrement des compétences facilitent l'imputation de ces politiques aux macro-institutions, telles l'Etat ou la Région. Beaucoup moins le Département et encore moins les structures intercommunales, tellement à la mode chez les élus, mais privées de toute légitimité élective au suffrage direct. Le vote n'exprime donc plus une histoire régionale où se confondaient des réalités de classe mais plutôt des demandes sectorielles et éparses sur tout le territoire d'une élection.

C'est ainsi que l'on peut entendre la notion de réajustement structurel qui a fait entrer la gauche tout entière, sans qu'elle en ait conscience, dans une déstabilisation durable et historique. Depuis 1997, la carte électorale législative du Parti Socialiste coöncide avec celle, non pas de la SFIO, mais du Parti Radical en 1928 (Salmon, 2001). C'est un indice parmi d'autres susceptible d'éclairer, sinon son destin, du moins le stade où en est arrivé la gauche méridionale.

\section{Les chasseurs dans lentre-deux}

Les chasseurs cultivent en Languedoc deux zones de votes: le littoral et l'arrière-pays. Dans les deux zones, il s'agit d'appuyer des convictions conservatrices, celles-là même qui veulent que le monde ne change pas, et rurales, qui cherchent aussi à préserver une certaine image du monde naturel. Ainsi, on retrouve dans les deux zones de force une composante rurale et solidariste, issue plutôt d'une socialisation de gauche, et une bourgeoisie foncière et agricole plutôt socialisée à droite. Les deux zones different finalement dans leurs préférences cynégétiques; l'une est l'aire de chasse des amateurs de gibier d'eau et de volatiles migrateurs, l'autre une terre de battue, de chasse au gros gibier. Les deux mondes cynégétiques sont proches, et s'interpénètrent facilement. La chasse constitue une réelle société, où les hommes se croisent, discutent, échanges des biens sauvages ou rares, gibier, champignons, vins, poissons... C'est enfin un univers où se partagent voyages, repas, souvenirs, au-delà des barrières politiques traditionnelles. Ces communautés peuvent se mobiliser fortement, et constituent, au-delà d'un poids électoral suffisant, de véritables pouvoirs de nuisance contre les jeux électoraux traditionnels. En 2001, l'un deux a gagné au suffrage universel direct un poste de conseiller général de l'Hérault. Les chasseurs ne font aucunement peur, et leurs cantons d'implantation majeure n'hésitent plus aujourd'hui à les placer devant les appareils départementaux traditionnels. Si le score de Jean Saint-Josse au $1^{\text {a }}$ tour des présidentiels révèle encore cette difficulté à intéresser lors des forts enjeux nationaux, plusieurs circonscriptions législatives pouvaient être le terrain d'une meilleure implantation. Ce ne fut pas le cas, hormis dans la $5^{\mathrm{e}}$ circonscription de 
l'Hérault, où le candidat CPNT, en $4^{c}$ position, totalisait près de $10 \%$ des voix à l'issue du premier tour. Là, les 5400 voix "chasseurs" du premier tour ont été l'objet de toutes les convoitises, et sont certainement la clé d'une victoire du candidat socialiste, que l'on donnait battu le 9 juin au soir. Partout ailleurs, les candidats CPNT n'ont pu passer la barre des $5 \%$. La nationalisation extrême des enjeux de ces législatives, les appels répétés, à gauche et à droite, au vote utile, ont réduit considérablement la surface électorale des formations "tribuniciennes".

\section{Itinéraires de la droite de 1986 aे 2002}

La droite languedocienne est-elle à un tournant stratégique? Cette question est bien sûr valable pour l'ensemble du territoire français. L'union réalisée des partis de droite, et la recomposition du camp entre un UMP pesant $80 \%$ du champ électoral de droite, et une UDF encore en peine d'exister nationalement partout, fait envisager, si l'alliance est durable, une recomposition complète des batailles électorales à venir. En Languedoc, cette recomposition prend trois particularités:

Tout d'abord, elle a éliminé du jeu l'UDF. La dernière personnalité forte de l'UDF, JeanPaul Alduy, sénateur des P. O. et maire de Perpignan, s'est rallié à l'UMP. Dans cette région qui n'a jamais été une terre centriste, la formation de François Bayrou n’a présenté que 2 candidats sur les 21 circonscriptions. L'un disputait en candidat unique de la droite une circonscription socialise audoise, l'autre, à Bagnols-sur-Cèze, tentait un duel de premier tour avec le candidat UMP.

Ensuite, la localisation des enjeux a contribué à unifier la droite avant l'UMP. Cette stratégie a permis la conquête de plusieurs villes moyennes en 2001. La droite languedo- cienne, essentiellement basée sur une alliance DL-RPR, s'est unie avant l'heure.

Il lui restait à surmonter un dernier handicap, troisième particularité de la région: le déficit de légitimité de son leader, Jacques Blanc, dans le champ politique national.

La nécessité de gagner, et la mainmise du Président de région sur les "lieutenants" locaux, ont facilité cette relégitimation de Blanc, pourtant toujours allié au FN et au MNR pour la conduite de sa politique régionale.

Aussi, si la droite languedocienne semble marcher au même rythme que la droite nationale, elle y vient par des chemins tortueux.

On peut lire l'histoire de la droite languedocienne récente comme la succession des stratégies à court terme mises en œuvre par celui qui s'est imposé comme son leader exclusif. Parti à la conquête de l'exécutif régional en 1986, Jacques Blanc n'a longtemps eu d'assises politiques qu'en Lozère, son bastion premier. Dans cette terre profondément ancrée d̀ droite, il a su, dans les années 80 , succéder aux dynasties notabiliaires familiales pour fonder la sienne. Si sa première victoire en 1986 lui a donné une tribune régionale, elle n'a pas en soi suffit à l'imposer comme leader régional, notamment parce qu'elle était - déjà - acquise avec le soutien du Front National. C'est dans la conquête même de cette alliance, et son issue, que Jacques Blanc a conquis ses véritables galons de leader. Ces proches ont théorisé ce moment comme celui de la stratégie de l'avalement: le travail essentiel du président de région fut de réintégrer dans sa majorité les notables locaux élus sous la bannière FN, jusqu'à vider le groupe frontiste à la région de son pouvoir de nuisance.

C'est que la tentation extrémiste et/ou séparatiste est depuis longtemps une composante de la droite languedocienne. Une droite qui n'est pas, historiquement, une droite 
républicaine (Négrier, 2001). Aussi ces dix dernières années ont-elles été marquées par deux types de stratégies: la reconquête des votes frontistes, et l'alliance avec les lepenistes, d'une part, la maîtrise plus ou moins contrôlée des expériences De Villiers, Pasqua, etc. Après la réussite de la stratégie de "l'avalement" (1986-1992), Les votes protestataires à droite ont considérablement nuit aux stratégies RPR et DL, Pasqua ayant même réuni sous sa bannière $15 \%$ des votes lors des élections européennes dernières. En 1998, Blanc et son équipe ont donc retenté une stratégie d'avalement des frontistes au sein de la majorité du conseil régional. Cette stratégie s'est soldée par un échec relatif: les frontistes, désormais séparés en deux groupes, n'ont connu aucune défection de leurs rangs vers les bancs de Jacques Blanc. Mais l'alliance n'a jamais été dénoncée pour autant.

Ce faisant, Blanc a semble-t-il compris la nécessité de changer de stratégie locale. Les élections municipales ont alors été l'occasion d'un autre tournant stratégique, l'invention d'une "droite de courtoisie", très proche du FN dans ses thématiques sécuritaires, se déclarant souvent apolitique. C'est cette stratégie qui a permis à la droite régionale de conquérir les villes moyennes du Languedoc.

La force du leadership de Blanc est de permettre d̀ ses lieutenants de conforter leurs positions, sans interférer vraiment dans les querelles intestines incessantes des majorités municipales. Les successions difficiles, les tornades locales, restent le plus souvent du domaine des élus directement impliqués. Seules les terres lozériennes et montpelliéraines font l'objet d'une surveillance constante, sur laquelle nous reviendrons.

Cette stratégie de proximité et de courtoisie a porté ses fruits aux municipales. Elle a failli mettre en péril l'ensemble de la droite au premier tour des présidentielles.
En effet, la proximité constante entre le discours des édiles de droite et les thèses du Front National permet vraisemblablement de capitaliser dans les échéances locales une partie des électeurs frontistes mouvants. Ce n'est plus le cas lors d'enjeux nationaux forts, où les regards lepenistes se tournent vers leur chef. Jacques Chirac était en troisième position au soir du premier tour, sur l'ensemble de la région, devancé de 80000 voix par JeanMarie Le Pen. La droite se retrouvait laminée, descendant à moins de $15 \%$ dans la plupart des villes, et notamment dans les villes conquises par une "droite de courtoisie".

Alors que le second tour permettait, nationalement, de remonter la droite, la région voyait les suffrages lepenistes augmenter encore, pour totaliser 10000 voix de plus que l'addition des voix Le Pen et Megret du premier tour. De façon caractéristique, les seuls vrais pôles de résistance à cet effondrement de la droite furent les villes dirigées par des maires ayant choisi de se distancier de cette stratégie du mimétisme: Perpignan, Saint-Gilles, etc. Dans le "coup de tonnerre" du premier tour des présidentielles, tout le péril d'une telle stratégie se laisse entrevoir.

Cela n'a pas eu d'échos. Les législatives, lancées par ce second tour inédit, ont remis le Languedoc-Roussillon dans la vague nationale. Jacques Blanc, promu par les appareils nationaux leader de l'UMP, présentait des candidats uniques dans la plupart des circonscriptions. La balance languedocienne, dont nous avons parlé précédemment, revenait à droite, et rééquilibrait la représentation législative du territoire, avec 14 députés de droite pour les 21 circonscriptions.

A cette occasion, l'implantation urbaine s'est trouvée largement confortée, ne laissant à la gauche que ces traditionnels bastions ruraux. Deux points méritent néanmoins d'être soulignés, à Montpellier et en Lozère. 
A Montpellier, Jacques Blanc cherche depuis longtemps à empêcher l'émergence de toute figure de droite qu'il ne contrôlerait pas. Les leaders successifs des groupes municipaux d'opposition ont ainsi connu leur lot de chausse-trappes et de coups bas internes, rendant impossible une structuration durable de l'opposition à Georges Frêche. Cela fait partie, très certainement, du partage du territoire auquel donne lieu l'incessant combat des deux hommes (Baraize, 2001). Cette fois encore, Jacques Blanc a su imposé un lieutenant fidèle face au président du groupe d'opposition municipale dans la bataille interne à la droite pour la candidature face au maire. Le nouveau lieutenant, Jacques Domergue, médecin des $\mathrm{CHU}$, a su profiter de l'usure du champion pour s'imposer des quelques voix au second tour. Georges Frêche battu, c'est une nouvelle victoire pour Jacques Blanc, qui l'a assumé comme telle. L'histoire dira s'il laissera ce nouveau challenger, qui n'a de cesse de lui proclamer sa fidélité, partir à la conquête de la capitale régionale en 2007.

Au milieu de ce flot de conquête, Jacques Blanc n'a qu'un souci : sa Lozère. Certes, la circonscription passée d̀ gauche en 1993 est revenue à droite. Mais le candidat de Jacques Blanc s'est fait battre par un dissident RPR, Jean Saint-Leger. L'incident devrait considérablement handicaper le candidat de Blanc, qui espérait mieux. Il traduit également un mouvement de fond : la conquête pied à pied de positions importantes par le RPR, contre les dynasties traditionnelles de la droite catholique lozérienne regroupée derrière Blanc. Ce n'est pas le leadership régional qui se joue ici, mais la recomposition d'une terre votant à $70 \%$ à droite, et la conquête du conseil général de la Lozère par des représentants d'une droite plus moderniste que les héritiers des "maitres de granit" (Pourcher, 1995).
Cela ne devrait néanmoins pas gâcher la victoire de Blanc, qui a vu plusieurs de ses vices-présidents à la région accéder à l'assemblée nationale, et dont les composantes territoriales les plus difficiles de sa majorité (le Gard, notamment), connaissent un début de remise en ordre.

Pourtant, si le repli de la gauche est net, et profite mécaniquement à la droite, il faudra, pour juger vraiment de la réussite de sa nouvelle stratégie, que la poursuite de l'union se fasse sans heurts au plan national d'une part, et que la confirmation ou non du repli des votes frontistes aux élections législatives ait des échos lors des autres scrutins.

Ce repli est il le reflet régional et mécanique de la "légitimation" de Jacques Chirac dans l'incarnation de la république, qui profiterait mécaniquement à l'UMP et à son leader Jacques Blanc? Ou traduit-elle plus profondément une restructuration de l'électorat d'extrême-droite?

\section{L'ambivalence de l'implan- tation de l'extrême-droite}

Le Languedoc-Roussillon témoigne à la fois, à l'occasion de ces deux scrutins, de tendances convergentes avec les constats établis à l'échelle nationale, et de caractéristiques qui lui sont, souvent depuis longtemps, singulières. Parmi les premières, on notera d'abord que le pari que certains faisaient sur l'extinction de ce courant politique à la faveur de sa scission de 1999 est en échec radical. Non seulement le FN parvient-il à accroître encore le nombre de ses suffrages d'une présidentielle à l'autre, mais la traduction de cette (somme toute légère) augmentation en voix en pourcentage de suffrages exprimés est-elle considérable: $24,9 \%$ (Le Pen + Mégret au premier tour 2002) contre $18,2 \%$ (Le Pen 1995). 
Ensuite, en regard du succès du FN, l'échec du MNR est, en Languedoc, comparable à sa déconfiture nationale. Alors qu'il comptait dans cette région quelques-uns de ses leaders de la première heure (notamment dans le Gard) et qu'il avait fait, plus que le FN, le pari d'une forme de notabilisation de ses représentants locaux, sa régression, même vis-à-vis des piteux scores des élections européennes de 1999, est globale. Dans tous les départements, son influence est groupusculaire. Il n'y a qu'en Lozère où le MNR fait légèrement mieux $(2,38 \%$ contre $2,25 \%)$ aux élections législatives qu'aux européennes de 1999. Mais encore est-ce en additionnant ses voix et celles de "Droit de chasse", qui en était l'émanation directe.

Le troisième phénomène convergent est la progression du FN dans les anciens bastions du PCF. Chaque élection semble faire basculer un nouveau terrain au profit de l'extrêmedroite. Ainsi le territoire anciennement minier d'Alès avait largement contenu, lors des précédentes législatives et régionales, l'influence de l'extrême-droite, grâce au maintien d'un relativement haut niveau de vote communiste. Cette fois, la chute de ce dernier va de pair avec une augmentation considérable des voix en faveur de J.-M. Le Pen. De même le PCF, après avoir perdu en 2001 l'une de ses rares municipalités de l'ouest héraultais, Vendres, voit-il le leader frontiste s'imposer largement en tête. Dans ce dernier cas, l'évolution des scores respectifs sur cinq ans montre une progression linéaire (de $17 \%$ vers $26 \%$ des suffrages exprimés) du vote $\mathrm{FN}$ et une régression de même type (de $32 \%$ vers $13 \%)$ du vote PC.

Enfin ce poids électoral croissant du FN ne lui permet toujours pas de peser significativement sur les élections nationales à deux tours, pour lesquelles le mode de scrutin constitue naturellement une assez forte contrainte. Sur la base des résultats du premier tour, le FN pouvait mécaniquement se maintenir dans 17 circoncriptions sur 21. En réalité, il n'aura pu le faire que dans 4: deux dans le Gard et deux dans l'Hérault. Le premier parti des élections présidentielles en Languedoc-Roussillon n'obtient aucun député. L'élection présidentielle, avec son cortège de personnalisation, de simplification est naturellement plus favorable à un parti fonctionnant sur le populisme charismatique de son chef que ne le sont des scrutins de circonscription, où jouent davantage l'enracinement politique, les réseaux notabiliaires et relais sociaux, ressources dont le FN ne bénéficie nullement.

Mais ces deux élections démontrent également le maintien d'une spécificité languedocienne, que l'on peut repérer à au moins trois niveaux.

Le premier est bien sûr celui de l'ampleur des scores obtenus par l'extrême-droite. Le "laboratoire" languedocien, que nous avions caractérisé dès 1998 (Alliès et alii., 1998, 1999) travaille régulièrement un territoire de plus en plus vaste. Jean-Marie Le Pen arrive en tête au premier tour dans la région, et l'extrême-droite cumule un score de $24,9 \%$ des suffrages exprimés. Ceci faisait alors du Languedoc-Roussillon sa troisième région française en influence électorale, derrière l'Alsace et Provence-Alpes-Côte d'Azur. Mais le phénomène sans doute le plus significatif est la progression en voix de J.-M. Le Pen entre les deux tours. Au second, celui-ci gagne en effet plus de 12000 électeurs sur le score cumulé Le Pen-Mégret du premier. Le Languedoc-Roussillon devient à cette occasion sa seconde région française (avec $24,46 \%$ des suffrages exprimés en faveur de Le Pen), derrière PACA $(27,69 \%)$.

Comment expliquer cet étalement languedocien de l'extrême-droite? Trois thèses, d'inégale pertinence, permettent 
d'en cerner la dynamique.

La première est historique. Elle fait reposer la spécificité languedocienne sur la présence, enracinée, d'un courant extrêmiste déjà repérable, par exemple, lors des premières élections présidentielles au suffrage universel de 1965 . Le vote Tixier-Vignancourt y avait déjà atteint le record (pour l'époque) de $11 \%$, soit six points au-dessus de la moyenne nationale. Mais ce premier "signal" n'a eu aucune postérité pendant 20 ans, et il a fallu attendre 1984 (européennes) et 1986 (régionales) pour voir resurgir cette tendance réputée enracinée. Pour donner crédit à cette thèse, il faut expliquer pourquoi le vote extrême émerge en tant que tel dans certaines périodes, pour demeurer latent dans d'autres. À cet égard, la thèse de Philippe Secondy (2001) mérite attention, car elle s'intéresse moins à la fin $\mathrm{du} \mathrm{XX}^{\mathrm{e}}$ siècle qu'à la structure spécifique de la droite depuis la fin du XIX ${ }^{e}$ siècle. Celleci est marquée par la faiblesse durable de l'orientation chrétienne-démocrate, qui a assuré, ailleurs, un ralliement plus net à la République. Les Croix-de-Feu du colonel De La Rocque, le Parti Social Français et le PPF de Doriot, y ont joué un rôle important. Le quotidien régional $L^{\prime} E c l a i r$, ancêtre du Midi-Libre, demeure, au moins dans sa rédaction, monarchiste jusqu'en 1944. Même si les courants nationaux (RPF, MRP) s'implantent sous la IV' République, la droite connaît en Languedoc des difficultés à s'inscrire dans la nouvelle donne sociale et politique. En témoigne l'influence qu'y détient le CNIP (Centre National des Indépendants et Paysans), un parti charnière d'une droite hostile à la politique modernisatrice de la $V^{e}$ République. La droite languedocienne peine à sortir d'une vision "conservatrice" de la politique et de la société. Elle est idéologiquement plus proche, dans sa génétique régionale, des valeurs extrêmes que dans bien d'autres régions (Négrier, op. cit.).

La seconde thèse prolonge la première dans l'actualité politique languedocienne. Elle s'articule sur les pratiques politiques contemporaines, et notamment sur la stratégie d'alliance Droite-extrême-droite qui a cours au Conseil Régional depuis 1986, avec une parenthèse arithmétique entre 1992 et 1998 . Incontestablement, l'enrôlement du FN dans la majorité a conféré à ce dernier une place dont il ne dispose que dans peu de régions, et surtout, avec une telle continuité. C'est d'ailleurs sur la droite que l'extrême-droite puise l'essentiel de ses réservoirs de voix ${ }^{1}$. Le berceau historique et idéologique a donc pour prolongement une reconnaissance d'un courant, comme partenaire de la part des dirigeants régionaux de la droite, comme comportement politique "normal" de la part des électeurs ${ }^{2}$. C'est sans doute la raison d'un étalement des scores du FN dans des territoires nouveaux, et d'une progression de celui-ci dans ses zones de force. Deux exemples localisés le démontrent:

(i) Le score lozérien (15,3\% au premier tour présidentiel 2002) est équivalent à celui du FN dans l'Hérault aux élections européennes de 1984, que beaucoup avaient alors considéré comme une anomalie. La banalisation du FN à un très haut niveau pour ce département rend compte de la porosité de la ligne de partage électorale entre droite et extrême-droite: la première, au premier tour, n'obtient que $37,9 \%$ des suffrages (Chirac + Madelin + Bayrou + Boutin), très loin des $55 \%$ de 1995.

(ii) La domination du FN dans la $6^{c}$ circonscription de Béziers au même scrutin: Jean-Marie Le Pen arrive en tête dans les 
dix-sept communes de la circonscription, avec un maximum de $35,59 \%$ a ValrasPlage et un minimum de $22,6 \%$ à Cazouls-les-Béziers. Le candidat du F.N. recueille plus de $30 \%$ dans huit communes (Valras, Sérignan, Maraussan, Lignan, Colombiers, Lieuran, Cers et Boujan), entre 28 et $30 \%$ dans six communes (Béziers $(28,98 \%)$, Portiragnes, Colombiers, Corneilhan, Lespignan et Sauvian) et moins de $27 \%$ dans trois communes (Cazouls, Bassan (26,19\%), Vendres $(26,62 \%)$. La caractéristique principale de toutes ces communes ce sont des zones périurbaines.

Les chiffres de l'extrême-droite dans l'ensemble des départements ( $c f$. tableau) démontrent, si besoin était, l'ampleur de cet étalement et de cette banalisation du vote frontiste, quand bien même celui-ci serait privé de toute "efficacité représentative" lors du passage aux élections législatives.

Le cas du biterrois nous amène à la troisième thèse, qui s'appuie sur la structure démographique de cette progression. Un vote littoral, urbain et périurbain, disionsnous en 1998 (Négrier, 1998). En dépit de son étalement régional, cette structure dominante demeure pertinente pour ces élections de 2002, avec une amplitude spécifique pour les territoires périurbains, ceux qui ont littéralement explosé démographiquement lors de cette dernière décennie. Or cette croissance, qui est due pour l'essentiel à une immigration intérieure (des autres régions françaises et des centres-villes languedociens), dessine la carte d'une certaine détresse de classe moyenne: perte des réseaux sociaux antérieurs au nouvel aménagement, difficulté d'insertion dans des communautés villageoises elles-mêmes perturbées par le changement de leur physionomie, focalisation sur le patrimoine individuel nouvellement acquis... etc. Le vote $\mathrm{FN}$ pourrait être qualifié de vote de l'angoisse pavillonnaire, à laquelle correspondent, sur ces mêmes zones, des chiffres convergents : hausse du taux de surendettement des ménages, taux de divorces supérieurs à la moyenne... La sensibilité au discours sécuritaire (offert par la droite et recueilli par l'extrême-droite) y est plus forte, de même que dans les communes littorales, où il s'adresse à une population plus âgée que la moyenne.

Enracinement, reconnaissance et étalement marquent donc la progression du FN en Languedoc-Roussillon. Pourtant, entre les deux tours et comme partout en France, la région semblait avoir donné de la voix, dans la rue, pour dénoncer cette dérive politique. 35000 manifestants à Montpellier, 3000 à Sète, et 5000 à Alès: des scores historiquement inédits, dépassant même parfois les records de mai 1968 ! Force est de constater que la "révolte de la jeunesse", telle qu'on l'a, sans doute hâtivement dénommée, a eu une faible traduction dans les urnes, et n'a pas empêché le Languedoc-Roussillon de demeurer une terre d'élection particulièrement stable pour le FN. 
les élections présidentielles et législatives de 2002 en L.R.

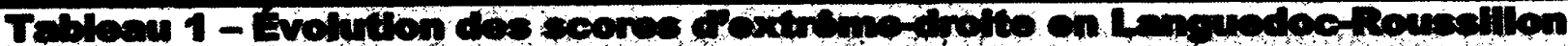

(on \% des suffreges exprimbs)

\begin{tabular}{|c|c|c|c|c|c|}
\hline & Aude & Gard & Hérault & Lozère & Pyrénées-o. \\
\hline Européennes 1984 & 8,5 & 12,7 & 15,3 & 6,7 & 15,9 \\
\hline Régionales 1986 & 6,1 & 14,8 & 14,1 & 5,1 & 17,8 \\
\hline Présidentielles 1988 & 13,7 & 20,6 & 19,9 & 11,6 & 20,5 \\
\hline Européennes 1989 & 10,0 & 14,8 & 15,9 & 8,0 & 18,7 \\
\hline Régionales 1992 & 11,6 & 18,2 & 18,3 & 5,9 & 22,6 \\
\hline Législatives 1993 & 11,8 & 17,8 & 14,9 & 5,6 & 18,3 \\
\hline Européennes 1994 & 8,8 & 13,8 & 13,3 & 6,2 & 14,8 \\
\hline Présidentielle 1995 & 13,6 & 20,3 & 19 & 9,6 & 19,5 \\
\hline Législatives 1997 & 14,4 & 21,3 & 19,3 & 8,3 & 21,2 \\
\hline Régionales 1998 & 13,2 & 21,9 & 17,3 & 5,7 & 17,9 \\
\hline Européennes 1999 & $\begin{array}{l}8,28 \\
\text { FN: } 5,27 \\
\text { MNR: } 3,01\end{array}$ & $\begin{array}{l}13,36 \\
\text { FN: } 7,61 \\
\text { MNR: } 5,75\end{array}$ & $\begin{array}{l}10,83 \\
\text { FN: } 6,09 \\
\text { MNR: } 4,74\end{array}$ & $\begin{array}{l}5,42 \\
F N: 3,17 \\
\text { MNR: } 2,25\end{array}$ & $\begin{array}{l}11,62 \\
F N: 7,18 \\
\text { MNR: } 4,44\end{array}$ \\
\hline Présidentielles 2002 & $\begin{array}{l}22,3 \\
\text { FN: } 19,8 \\
\text { MNR: } 2,5\end{array}$ & $\begin{array}{l}28 \\
F N: 24,9 \\
M N R: 3,1\end{array}$ & $\begin{array}{l}25,4 \\
\text { FN: } 23 \\
\text { MNR: } 2,4\end{array}$ & $\begin{array}{l}15,3 \\
\text { FN: } 13,6 \\
\text { MNR: } 1,7\end{array}$ & $\begin{array}{l}23,3 \\
F N: 20,9 \\
\text { MNR: } 2,4\end{array}$ \\
\hline Législatives 2002* & $\begin{array}{l}16,16 \\
\text { FN: } 14,03 \\
\text { MNR: } 2,13\end{array}$ & $\begin{array}{l}20,65 \\
\text { FN: } 18,34 \\
\text { MNR: } 2,31\end{array}$ & $\begin{array}{l}18,19 \\
\text { FN: } 16,74 \\
\text { MNR: } 1,45\end{array}$ & $\begin{array}{l}7,71 \\
\text { FN: } 5,33 \\
\text { MNR: } 2,38\end{array}$ & $\begin{array}{l}17,68 \\
\text { FN: } 16,6 \\
\text { MNR: } 1,08\end{array}$ \\
\hline
\end{tabular}

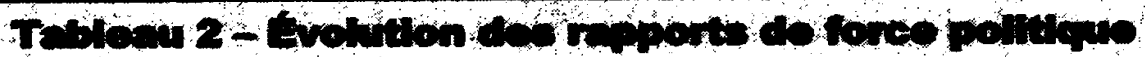

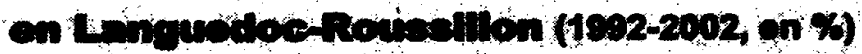

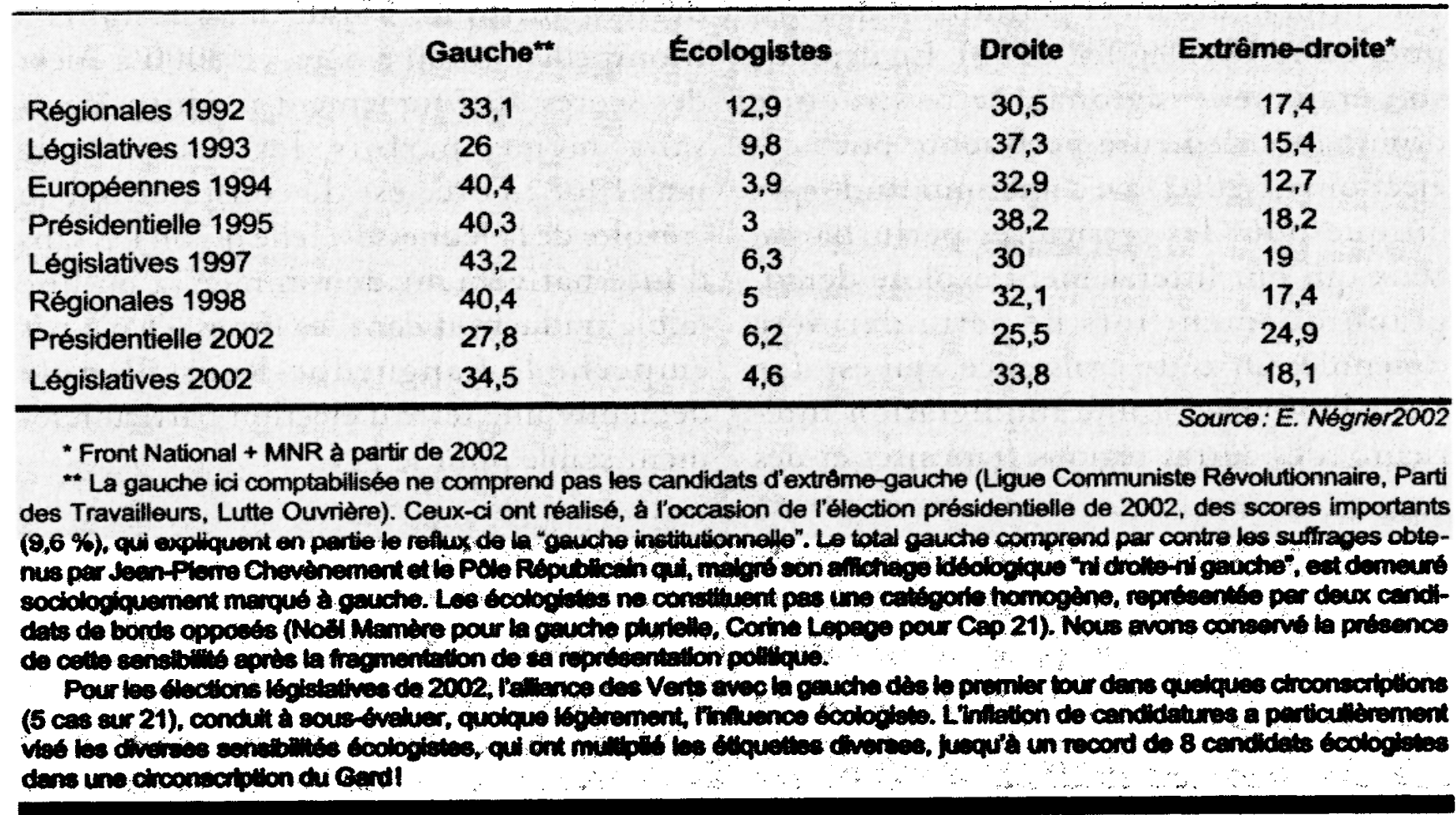




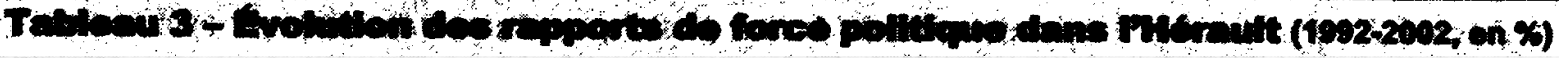

\begin{tabular}{lcccc}
\hline & Gauche & Ecologistes & Droite & Extrême-droite* \\
Régionales 1992 & 28 & 15,2 & 31,5 & 18,3 \\
Législatives 1993 & 35,7 & 10,8 & 36,9 & 14,9 \\
Européennes 1994 & 39,5 & 4,23 & 2,7 & 13,3 \\
Présidentielle 1995 & 39,6 & 3,3 & 38 & 19 \\
Législatives 1997 & 43,3 & 6,8 & 28,5 & 19,3 \\
Régionales 1998 & 43 & 4,8 & 34 & 17,3 \\
Présidentielle 2002 & 27,5 & 7,1 & 24,9 & 25,4 \\
Législatives 2002 & 31,8 & 7,2 & 32,8 & 18,2 \\
\hline
\end{tabular}

Tabloau 4 - Livolution des reppoits ib fores pollitique on Loxdro (1992-2002; on x)

\begin{tabular}{lcccc}
\hline & Gauche & Ecologistes & Drolte & Extrême-droite* \\
Régionales 1992 & 23,7 & 7,1 & 63,3 & 5,9 \\
Législatives 1993 & 20,6 & 6,5 & 67,3 & 5,6 \\
Européennes 1994 & 28,5 & 3,8 & 51,2 & 6,2 \\
Présidentielle 1995 & 31,7 & 3,2 & 55,1 & 9,6 \\
Législatives 1997 & 33,8 & 6,5 & 51,5 & 8,3 \\
Régionales 1998 & 27,5 & 2,3 & 64,5 & 5,7 \\
Présidentielle 2002 & 22,8 & 63 & 7,9 & 15,3 \\
Législatives 2002 & 31,1 & 3,15 & 1,5 & 7,7 \\
\hline
\end{tabular}

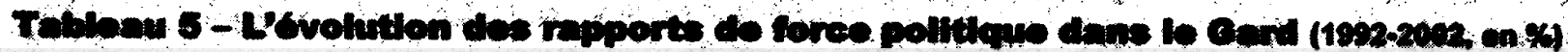

\begin{tabular}{lllll}
\hline & Gauche & Ecologistes & Drolte & Extréme-droite* \\
Régionales 1992 & 40,4 & 11,6 & 24,9 & 18,2 \\
Législatives 1993 & 38 & 9,4 & 33,2 & 17,8 \\
Européennes 1994 & 40,9 & 4,1 & 31,3 & 13,8 \\
Présidentielle 1995 & 39,5 & 3 & 37 & 20,3 \\
Législatives 1997 & 41,4 & 5,8 & 29,4 & 21,3 \\
Régionales 1998 & 38,3 & 4,6 & 27,9 & 21,9 \\
Présidentielle 2002 & 26 & 5,8 & 25,5 & 28 \\
Législatives 2002 & 33,6 & 3,6 & 36,2 & 20,6 \\
\hline
\end{tabular}

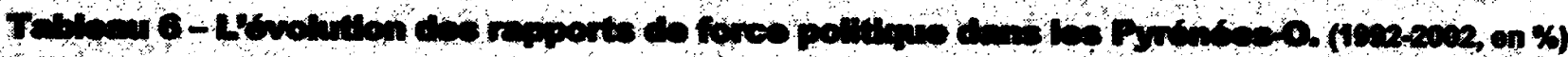

\begin{tabular}{lcclc}
\hline & Gauche & Ecologistes & Droite & Extrême-droite* \\
Régionales 1992 & 27,4 & 12,6 & 28 & 22,6 \\
Législatives 1993 & 31,7 & 9,1 & 39,2 & 18,3 \\
Européennes 1994 & 38,3 & 3,7 & 32,7 & 14,8 \\
Présidentielle 1995 & 38,6 & 2,7 & 39,1 & 19,5 \\
Législatives 1997 & 41,2 & 5,6 & 31,1 & 21,2 \\
Régionales 1998 & 38,8 & 5,2 & 27,6 & 17,9 \\
Présidentielle 2002 & 28,2 & 5,7 & 27 & 23,3 \\
Législatives 2002 & 37,1 & 2,3 & 32,4 & 17,7 \\
\hline
\end{tabular}

- Front National + MNR a partir de 2002 
les élections présidentielles et législatives de 2002 en L.R.

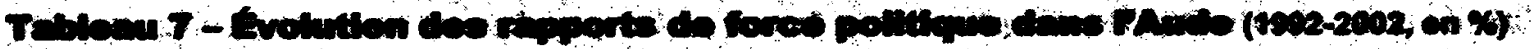

\section{Extreme-droite*}

Régionales 1992

Législatives 1993

Européennes 1994

Présidentielle 1995

Législatives 1997

Régionales 1998

Présidentielle 2002

Législatives 2002

\section{Gauche}

41

42,4

46,8

47,7

50,4

43,1

32,7

40,7

\section{Ecologistes}

31,6

35,4

32

35,8

28

30,7

22,5

28,9

\section{Droite}

11,6

11,8

8,8

13,6

14,4

13,2

22,3

16,2

Source: E. Negrier 2002

* Front National + MNR a partir de 2002

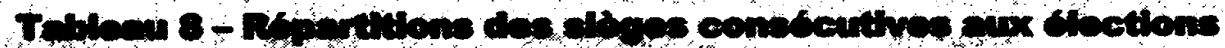

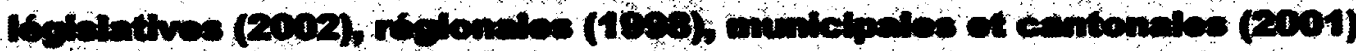

\begin{tabular}{|c|c|c|c|c|c|c|c|c|c|c|}
\hline & PC & $\begin{array}{c}\text { PS } \\
\text { et div } \\
\text { Gauche }\end{array}$ & Verts & $\begin{array}{l}\text { UDFI } \\
\text { DL }\end{array}$ & RPR & DVD & FN & Chass & & $\begin{array}{c}\text { Rapport } \\
\text { Gauchel } \\
\text { Droite }\end{array}$ \\
\hline Députés & 1 & 6 & & $\begin{array}{r}13 \\
(\mathrm{UMP})\end{array}$ & & & 1 & & & $7 / 14$ \\
\hline Sénateurs & & 8 & & 2 & 1 & & & & & $8 / 3$ \\
\hline C. Régional & 8 & 23 & & 10 & 11 & & 1 & 13 & 1 & $31 / 22$ \\
\hline C. Général. Aude & 2 & 27 & & & 5 & & 1 & & & $29 / 6$ \\
\hline C. Genéral Gard & 8 & 23 & & 7 & 5 & & 3 & & & $31 / 15$ \\
\hline C. Général Hérault & 4 & 33 & 1 & 3 & 4 & & 3 & & 1 & $38 / 10$ \\
\hline C. Général Lozère & 1 & 5 & & 7 & 3 & & 9 & & & $6 / 19$ \\
\hline C. Général P.O. & 5 & 15 & & 5 & 2 & & 4 & & & $20 / 11$ \\
\hline $\begin{array}{l}\text { Villes de plus de } \\
10000 \text { habitants }\end{array}$ & & 6 & & 6 & 3 & & 6 & & & $6 / 15$ \\
\hline
\end{tabular}




\section{Notes}

1. Avec un cas particulier dans les Pyrénées-Orientales, département généralement favorable aux candidats d'extrême-droite. La relative modestie de son score au premier tour, là où plusieurs composantes de la droite étaient en lice, va de pair avec la plus forte augmentation des voix Le Pen au second: + 9357 voix, soit l'essentiel de la progression régionale. Ceci s'explique sans doute par la forte présence d'une culture rapatriée qui, orientée à droite, conserve les plus vives préventions à l'Egard des candidats gaullistes.

2. Au contraire de l'Alsace, où, après un triomphe au premier tour, l'extrême-droite chute. Ce phénomène semble s'expliquer par la mobilisation des "forces morales" alsaciennes, parmi lesquelles les Eglises, entre les deux tours. De telles forces semblent ne pas avoir de poids dans les mondes sociaux de la droite languedocienne.

\section{$\approx$ \\ Références}

Alliès P., "Un midi en voie de banalisation politique?", Pôle Sud, n² 2, 1995.

Alliès P., Baraize F., Cheylan J.-P., Genieys W., Negrier E., "Les élections régionales du 15 mars 1998 en Languedoc-Roussillon. Une nouvelle singularité politique?", Pôle Sud, n 8, mai 1998.

Alliès P., Baraize F., Negrier E., Secondy P., "Les élections européennes du 13 juin 1999 en Languedoc-Roussillon: de la nationalisation du vote à la régionalisation des particularismes", Pôle Such nº 11, 1999.

Baraize F., "Montpellier, y'a-t-il un challenger dans la ville?", in Negrier E. (dir.), Les maîtres du Sud, Golias, 2001.

Martin P., Comprendre les évolutions electorales, Paris, Presses de Sciences Po, 2000.

Négrier E., Les maîtres du Sud, Golias, 2001.

Négrier Emmanuel, "Territoire, leadership et société: Georges Frêche et Montpellier", Sciences de la sociêté, n 53, 2001.

Négrier E., "Languedoc-Roussillon: le résistible établissement", Limes, mai 1998.

Pourcher Y., Les man̂tres de granit: les notables de Lozère du XVII siècle à nos jours, Paris, Plon, 1995.

Salmon F., Atlas electoral de la France, Paris, Seuil, 2001.

Secondy P., La droite extrême dans l'Hérault (1890-1944). Sociologie historique d'une configuration politique, Thèse de science politique, Université de Montpellier I, 2001. 